\title{
Modeling, Stability, Synchronization, and Chaos and Their Applications to Complex Systems
}

\author{
R. Yamapi, ${ }^{1}$ G. Filatrella, ${ }^{2}$ M. A. Aziz-Alaoui, ${ }^{3}$ and H. G. Enjieu Kadji ${ }^{4}$ \\ ${ }^{1}$ Department of Physics, Faculty of Sciences, University of Douala, P.O. Box 24 157, Douala, Cameroon \\ ${ }^{2}$ Dipartimento di Scienze Biologiche ed Ambientali, Università del Sannio, Via Port'Arsa 11, 82100 Benevento, Italy \\ ${ }^{3}$ Laboratoire de Mathématiques Appliquées, Université du Le Havre, 25 rue Ph. Lebon, BP 540, 76600 Le Havre Cedex, France \\ ${ }^{4}$ Monell Chemical Senses Center, 3500 Market Street, Philadelphia, PA 19104, USA
}

Correspondence should be addressed to R. Yamapi; ryamapi@yahoo.fr

Received 15 January 2014; Accepted 15 January 2014; Published 27 February 2014

Copyright (C) 2014 R. Yamapi et al. This is an open access article distributed under the Creative Commons Attribution License, which permits unrestricted use, distribution, and reproduction in any medium, provided the original work is properly cited.

Issues related to chaotic dynamics, stability of oscillations, and their synchronization are widespread in nature, appearing ubiquitously in physics, chemistry, biology, engineering, and social science. Furthermore, potential applications in delicate areas, for instance, secure communication where transmitter and receiver are synchronized, explain the growing interest devoted to modeling and understanding.

In the current trend, collaborations and interdisciplinary researches are essential keys for mathematicians, chemists, physicists, biologists, and engineers to successfully investigate dynamical state in complex systems. This special issue addresses modeling, chaos, and synchronization processes in complex systems, based upon hypothesis or data from physics, chemistry, biophysics, and neuroscience. From 17 submissions, 5 papers are published in this special issue. Each paper was reviewed by at least two referees and revised accordingly by the authors. The themes include adaptive synchronization, stochastic chaotic systems, effective synchronization, exponential feedback coupling, distributed reduced-order observer-based protocol, nonlinear filtering, chaos synchronization, stability analysis, and control.

When it comes to synchronizing oscillations, different strategies have been investigated such as effective synchronization, adaptive synchronization, inverse synchronization, phase synchronization, and antiphase synchronization. This diversity of schemes is necessary because of the variety of systems under consideration. In Z. Wang et al.s paper, an adaptive sliding mode controller is designed to realize the asymptotical synchronization in mean squares and the almost surely synchronization for two different stochastic chaotic systems, one with unknown parameters and another with uncertain terms. The robustness and efficiency of the technique are checked out using the Lorenz-Chen and ChenLu systems.

The exponential function-based nonlinear controller can also be utilized to achieve effective synchronization between two drive-response systems surrounded by the random perturbations. Such a technique was considered in P. Louodop et al's paper to obtain a new optimized controller which can lead to a much faster synchronization than the one based upon fixed feedback gain. Moreover, the new controller is easy to implement, for it does not need adaptation algorithm and it is based on a simple electrical system.

In the master-slave system configuration, chaotic synchronization can be effectively preserved via nonlinear filtering process. The synchronization phenomenon can occur in nonlinear interconnected structures that could have some useful applications for some chaotic systems in filtering waves. An example of application is the analysis of wave, originated from earthquakes and tsunamis inasmuch; they can generate resonance at particular frequencies. Such systems have been considered and investigated in J. S. González-Salas et al.'s paper.

The stability analysis and control of chaotic oscillations are also of great interest because of their impact in building up practical applications as those addressed in G. Zhou 
et al.'s paper. Authors have considered in a new smooth Chua's system whose chaotic features are confirmed by the Lyapunov exponents. A linear controller and an adaptive controller are, respectively, proposed to globally synchronize two identical smooth Chua's systems estimated errors of uncertain parameters converge to zero as time increases.

When it comes to coordination control of multiagent systems, consensus problem is one of the most basic issues for the environment information changes and the communication links between agents may become unreliable. This particular matter concern is addressed in Y. Zhang et al.s paper who have investigated a group of agents with highdimensional linear coupling dynamics with an undirected switching interaction scheme.

Together, these papers represent an insightful investigation into the state of art in these majors' interdisciplinary topics. We hope this special issue captures the attention of the peers. We would like to acknowledge our gratitude to all the authors and reviewers who have made this special issue possible.

R. Yamapi

G. Filatrella

M. A. Aziz-Alaoui

H. G. Enjieu Kadji 


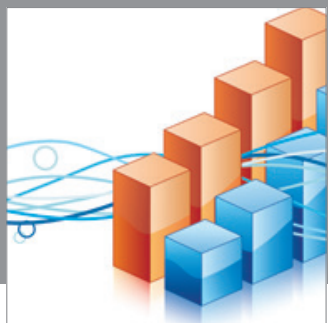

Advances in

Operations Research

mansans

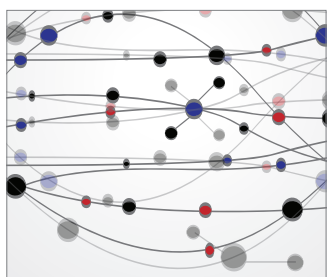

The Scientific World Journal
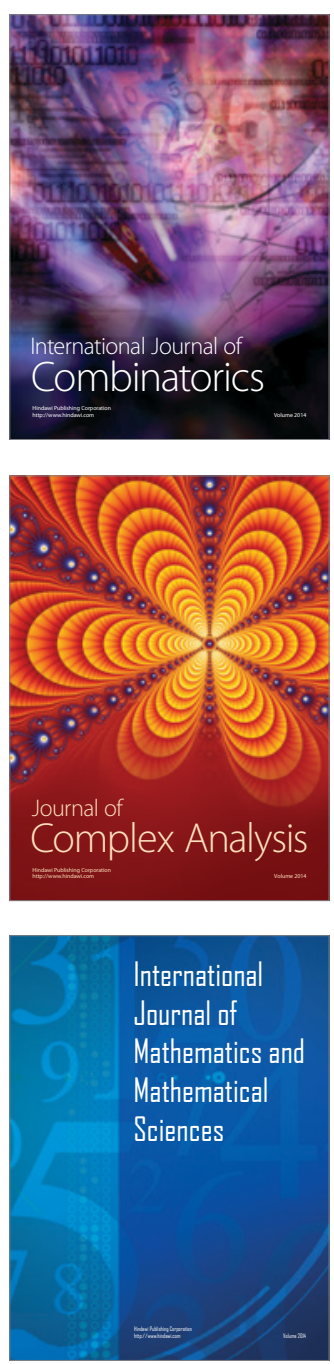
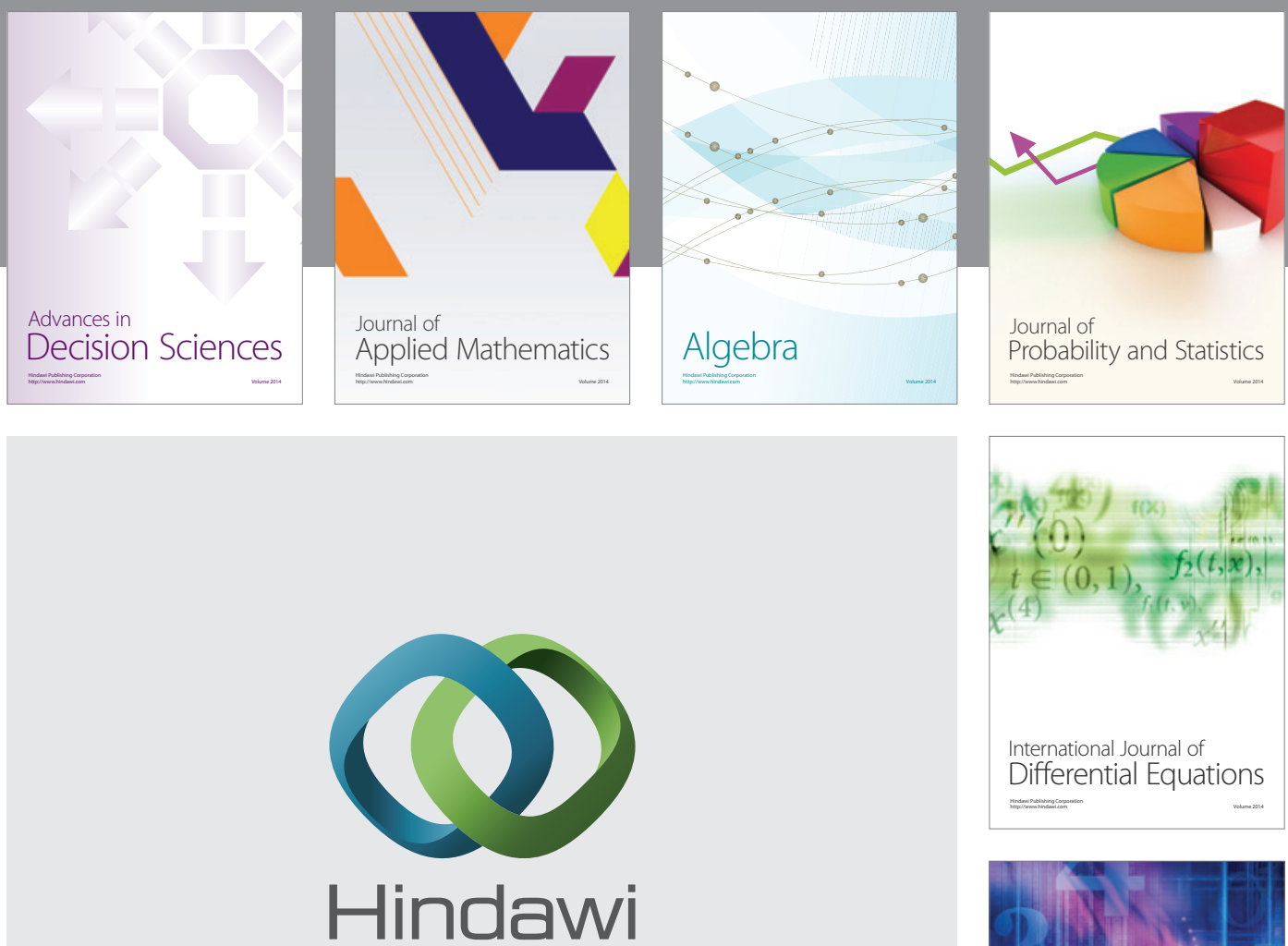

Submit your manuscripts at http://www.hindawi.com
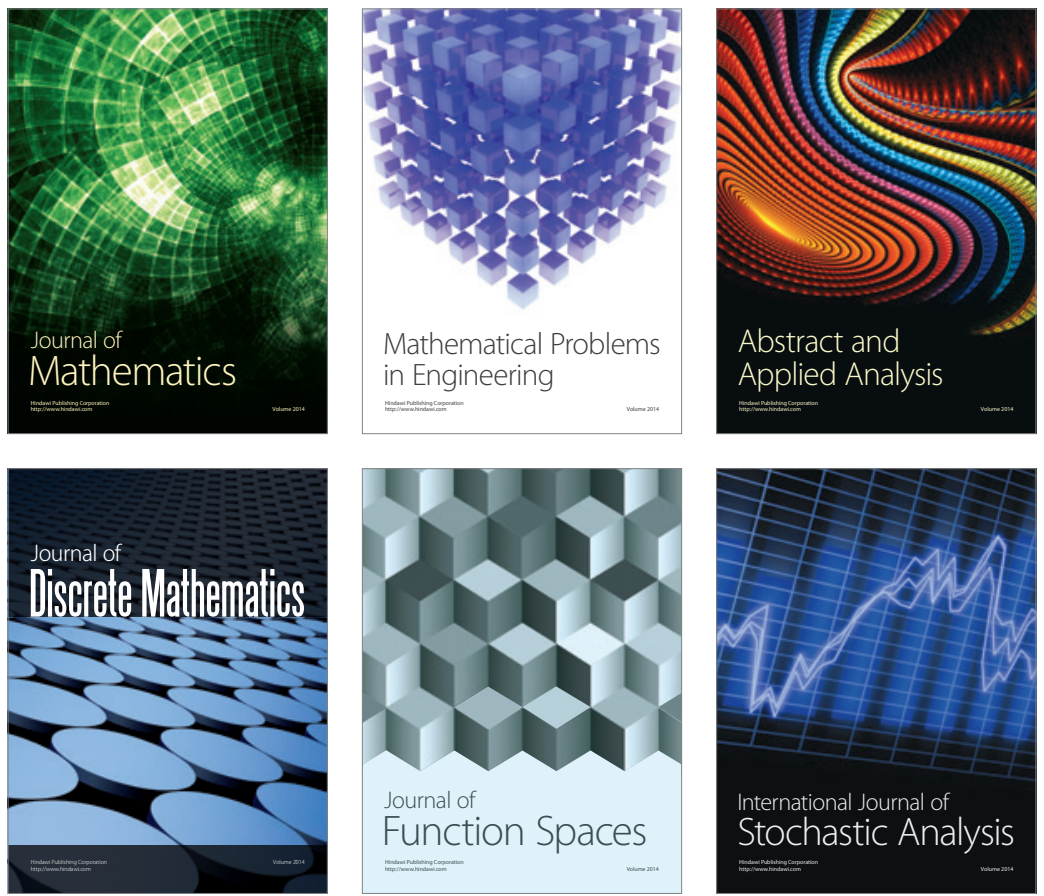

Journal of

Function Spaces

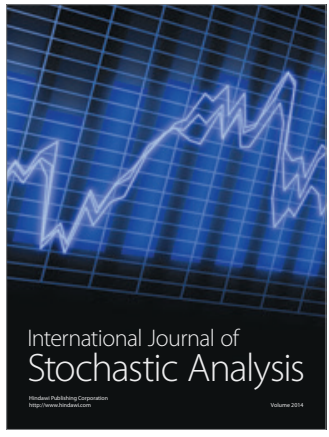

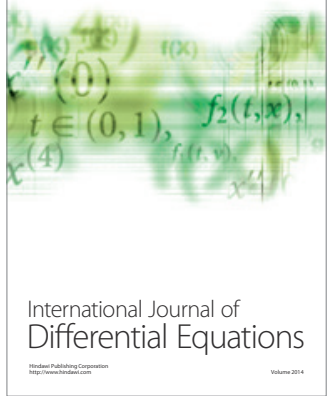
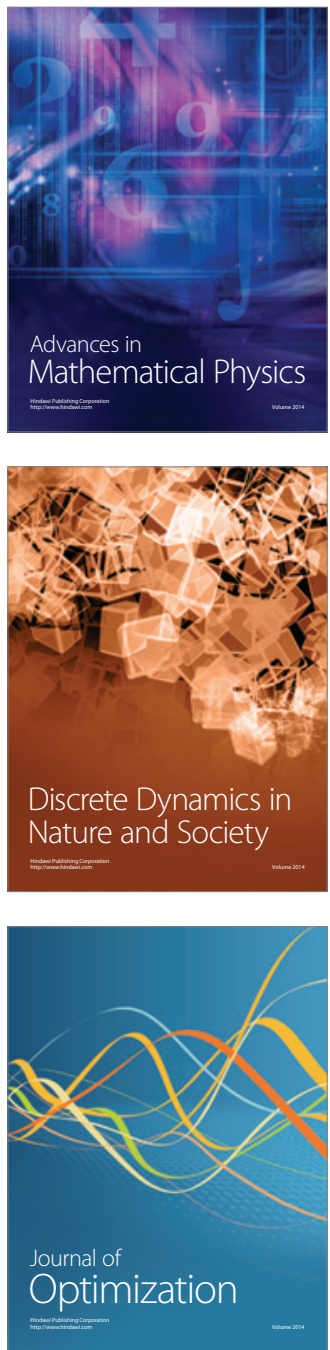